\title{
Die Induktion von Schülerkrisen durch Lehrpersonen. Professionalisiertes Lehrer/innenhandeln zwischen strukturtheoretischer Anforderungslogik und praxeologisch-wissenssoziologisch fundierter Rekonstruktion
}

\author{
Jan-Hendrik Hinzke $(D$
}

Angenommen: 2. März 2020 / Online publiziert: 12. März 2020

(C) Der/die Autor(en) 2020

Zusammenfassung Im Artikel wird das Thema der Induktion von Schülerkrisen durch Lehrpersonen aus zwei Perspektiven betrachtet. Erstens wird herausgearbeitet, was unter strukturtheoretischer Perspektive unter Krisen und Kriseninduktion verstanden wird. Dabei zeigt sich, dass Kriseninduktion im strukturtheoretischen Professionsansatz als Element professionalisierten Lehrerhandelns in den Blick gerät, das mit bestimmten Erwartungen verbunden und als Teil einer Anforderungslogik an Lehrpersonen herangetragen wird. Zweitens werden Ergebnisse einer explorativ angelegten, rekonstruktiven Interviewstudie präsentiert, der eine praxeologischwissenssoziologische Perspektive zugrunde liegt. In der Interpretation der Lehrer/ inneninterviews mit der Dokumentarischen Methode wird deutlich, dass die Induktion von Schülerkrisen voraussetzungsreich ist. Die Arbeit an der Kriseninduktion ist in jenen typischen Orientierungen verankert, die für Lehrpersonen in ihrem Berufsalltag leitend sind. Sie basiert auf einer spezifischen Relation von Normen und Common Sense-Theorien, von Orientierungsrahmen im engeren Sinne sowie von Orientierungsrahmen im weiteren Sinne. Aufbauend auf diesen beiden Perspektiven wird diskutiert, inwiefern die Interpretationsergebnisse als Ausdruck professionalisierten Lehrer/innenhandelns gedeutet werden können und welcher weitere Forschungsbedarf sich abzeichnet. Insgesamt betrachtet verfolgt der Artikel das Anliegen, dadurch einen Beitrag zum Diskurs um Professionalität in Bezug auf den Lehrer/ innenberuf zu leisten, dass strukturtheoretische Anforderungslogik an und praxeologisch-wissenssoziologisch fundierte Rekonstruktion von Lehrer/innenhandeln in Bezug zueinander gesetzt werden.

\footnotetext{
Dr. J.-H. Hinzke $(\bowtie)$

Wissenschaftliche Einrichtung Oberstufen-Kolleg, Fakultät für Erziehungswissenschaft, Universität Bielefeld, Universitätsstraße 23, 33615 Bielefeld, Deutschland

E-Mail: jan-hendrik.hinzke@uni-bielefeld.de
} 
Schlüsselwörter Kriseninduktion · Schülerkrisen · Lehrer/innenprofessionalität · Strukturtheoretischer Professionsansatz $\cdot$ Rekonstruktive Forschung ·

Dokumentarische Methode

\title{
Induction of crises of students by teachers. Professional teaching between structural theoretical demands and reconstruction based on the praxeological sociology of knowledge
}

\begin{abstract}
The article considers how teachers induct crises of students from two perspectives. Firstly, the understanding of crises and the induction of crises will be presented from a structural theoretical perspective. It becomes evident that the induction of crises is understood as part of professional teaching. It is connected with certain expectations and part of demands addressed to teachers. Secondly, results of an explorative reconstructive interview study with teachers, based on the perspective of the praxeological sociology of knowledge, will be shown. For data interpretation the documentary method is used. It becomes clear that inducting crises of students requires the fulfillment of preconditions. The induction of crises is embedded in those orientations that structure the everyday-work of teachers. It is based on a specific relation of norms and common-sense-theories on the one hand, of frames of orientations in a narrower sense on the other hand. This relation is framed by a frame of orientation in a wider sense. Based on these two perspectives it will be discussed how the results can be interpreted as an expression of professional teaching and which further research needs occur. In total, the article aims at contributing to the discourse on teacher professionalism by relating structural theoretical demands and reconstruction based on the praxeological sociology of knowledge.
\end{abstract}

Keywords Induction of crises - Crises of students - Teacher professionalism • Structural theoretical approach on teacher professionalism $\cdot$ Reconstructive research $\cdot$ Documentary method

Im deutschsprachigen Diskurs um Professionalität und Professionalisierung von Lehrpersonen erweisen sich drei „Bestimmungsansätze“ (Terhart 2011, S. 205) als dominant: kompetenztheoretischer, strukturtheoretischer und berufsbiographischer Bestimmungsansatz (Bonnet und Hericks 2014). Wenngleich in den letzten Jahren Brückenschläge zwischen diesen Ansätzen vorgenommen wurden (etwa Combe und Paseka 2012), lässt sich das Thema der Induktion von Schülerkrisen durch Lehrpersonen relativ eindeutig dem strukturtheoretischen Bestimmungsansatz zuordnen. Zwar ließe sich diskutieren, inwiefern mit einer so verstandenen Kriseninduktion nicht etwas Ähnliches wie mit der in kompetenztheoretischen Beiträgen verhandelten kognitiven Aktivierung (etwa Kunter et al. 2011) erreicht werden soll, doch nimmt die Induktion von Schülerkrisen nur im strukturtheoretischen Bestimmungsansatz eine zentrale Rolle ein. ${ }^{1}$

\footnotetext{
1 Zur besseren Lesbarkeit wird bei Komposita wie „Schülerkrisen“ und „Lehrerprofessionalität“ das generische Maskulinum genutzt.
} 
Diese Zentralstellung ist in einem ersten Zugriff insofern plausibel, als Krisen im strukturtheoretischen Verständnis eine ambivalente Konstellation darstellen, in der einerseits Altes zu einem Ende kommt, sich andererseits Neues entwickeln kann (Oevermann 1991). Die damit angesprochene Dialektik von Krise und Routine fasst Oevermann (1996, S. 75) wie folgt: ,[B]ezogen auf Routinen bedeuten Krisen deren Scheitern und damit ein manifestes Wieder-Öffnen der Zukunft, wohingegen Routinen immer die Schließung einer ursprünglichen Krise darstellen und insofern material aus dieser hervorgehen“. Angesichts der gescheiterten Routine gilt es in der Krise ,ins Offene hinein auf Verdacht“ (Oevermann 1991, S. 297), d.h. ohne vorliegende Begründungen, Entscheidungen zu treffen.

Das mit Krisen verbundene Potential für die Entstehung von Neuem wird in verschiedenen Lern- und Bildungstheorien thematisiert. Combe und Gebhard (2012) etwa sehen Irritationen bzw. Krisen als Herausforderung von Verstehen in der Schule an. Die Krise besteht darin, dass Lernende bei der Auseinandersetzung mit einem Unterrichtsinhalt eine Differenz zwischen ihrer Alltagsperspektive und der Sachperspektive erfahren (ebd., S. 7). Nach Koller (2012) ergeben sich nicht nur, aber potentiell auch in der Schule Krisen, wenn ,Menschen mit neuen Problemlagen konfrontiert werden, für deren Bewältigung die Figuren ihres bisherigen Welt- und Selbstverhältnisses nicht mehr ausreichen“ (ebd., S. 16). Krisen werden hier als Anlässe für biographisch relevante, transformatorische Bildungsprozesse betrachtet (ebd., S. $71 \mathrm{ff}$. ).

Diese Perspektiven aufgreifend wurde in den letzten Jahren von verschiedenen Autor/innen herausgestellt, dass die Induktion von Schülerkrisen Bestandteil der Professionalität von Lehrpersonen sei. ${ }^{2}$ Bonnet und Hericks (2014, S. 5) schreiben etwa, dass Lehrpersonen durch die Vermittlung von Wissen und Kompetenzen Krisen von Lernenden nicht nur lösen, sondern ,zuallererst“ „,auslösen“ können. Combe et al. (2018, S. 72) fokussieren hingegen das Potential von Kriseninduktion für den Umgang mit Kontingenz im Lehrerhandeln: „Um eine Auseinandersetzung des Ich mit der Welt [...] nachhaltig und prospektiv einzuleiten, müssen Ungewissheiten aktiv produziert sowie Krisen aufgegriffen und provoziert werden“.

Erstaunlich ist, dass es keine empirische Studie gibt, die im Kern dem Thema der Induktion von Schülerkrisen durch Lehrpersonen nachgeht. Studien aus dem Bereich der Unterrichts- (etwa Gruschka 2010) und der Ungewissheitsforschung (etwa Paseka und Schrittesser 2018) zeigen indes, dass Lehrpersonen im Unterricht eher um schnelle Schließungen von Kontingenz bemüht sind als dass sie Öffnungen - Schülerkrisen wären ebensolche - zulassen (auch Helsper 2014).

In dieser Studie wird das Ziel verfolgt, Bedingungen der Induktion von Schülerkrisen zu erschließen. Dazu wird der Forschungsfrage nachgegangen, welche handlungsleitenden Wissensbestände einer solchen Kriseninduktion durch Lehrpersonen zugrunde liegen. Nach einer Auseinandersetzung mit der Anforderung der

\footnotetext{
2 Die Begrifflichkeiten changieren dabei. Es finden sich u. a. „Kriseninduktion“ (etwa Combe und Gebhard 2012, S. 14), „Krisenauslösung“ (etwa Bonnet und Hericks 2014, S. 5) und „Kriseninitiation“ (etwa Helsper 2014, S. 219), wobei die Unterschiede zwischen den Begriffen nicht klar werden. In diesem Artikel wird durchgehend der Begriff „Kriseninduktion“ verwendet. Dadurch soll betont werden, dass Lehrpersonen Krisen von Schüler/innen nicht herstellen, sondern allenfalls anregen können (s. Kap. 1).
} 
Kriseninduktion im strukturtheoretischen Bestimmungsansatz (Kap. 1) und aufbauend auf einer Sichtung vorliegender Studien (Kap. 2) werden die Forschungsfrage und das methodische Design der mit der Dokumentarischen Methode operierenden, explorativen Studie, in der Lehrpersonen interviewt wurden, entfaltet (Kap. 3). Im Ergebniskapitel steht jener Fall im Zentrum, bei dem sich als einziger im Sample die Arbeit an einer Induktion von Schülerkrisen rekonstruieren ließ. Dargelegt wird, durch welche typischen handlungsleitenden Wissensbestände bzw. Orientierungen diese Arbeit an der Kriseninduktion strukturiert wird (Kap. 4). Abschließend wird diskutiert, wie Kriseninduktion aus praxeologisch-wissenssoziologischer Perspektive, d. h. jenseits der Erfüllung einer an den Lehrerberuf herangetragenen, normativen Anforderung, als Ausdruck von Professionalität gefasst werden kann und welche weiteren Forschungsfragen sich abzeichnen (Kap. 5).

\section{Kriseninduktion im strukturtheoretischen Professionsansatz}

Im strukturtheoretischen Professionsansatz stellt die Induktion von Schülerkrisen einen wesentlichen Teil einer an die Lehrertätigkeit herangetragenen Anforderungslogik dar.

Grundlegend sind die Ausführungen Oevermanns, nach denen professionalisiertes Handeln dort ansetzt, wo eine Lebenspraxis ,zur selbständigen Krisenbewältigung überfordert ist“ (Oevermann 2002, S. 26). Die „Struktur des Handlungsproblems“ (ebd., S. 20) besteht in einer stellvertretend für die Klient/innen durchgeführten Krisendeutung (ebd., S. 30) bzw. -bewältigung (ebd., S. 23). Dadurch bezeichnet Oevermann (ebd., S. 35) den Pädagogen „der Strukturlogik seines Handelns nach“ als „Geburtshelfer im Prozess der Erzeugung des Neuen“.

Im Lehrerhandeln geht es dabei weniger um die Wiederherstellung beschädigter Autonomie denn um die Unterstützung von Schüler/innen bei der Entwicklung von Autonomie (Oevermann 1996). Angesichts dessen fasst Oevermann pädagogisches Handeln unter dem Focus therapeutischen Handelns. Dessen Professionalisierungsbedürftigkeit ergibt sich daraus, dass es sich um ein nicht-standardisierbares ,prophylaktisches Handeln im Hinblick auf sein Potential der Weichenstellung der Biographie von Schülern in Richtung auf psychosoziale Normalität oder Pathologie“ (ebd., S. 149) handelt. In einem späteren Artikel nimmt Oevermann (2002) stärker die ,pädagogische Dienstleistung der Wissensvermittlung“ (ebd., S. 38) in ihrem „Krisencharakter“ (ebd., S. 37) in den Blick. Wissensvermittlung und -aneignung betrachtet er als einen „mäeutisch-sokratischen“ Prozess, ,in dem der Lernende durch das in sich krisenhafte Problematisieren von Voraussetzungen dazu provoziert wird, Fragen aufzuwerfen und sich ihnen so zu stellen, dass er auf dem Wege des Suchens einer Antwort so weit wie möglich selbst voran kommt" (ebd., S. 48).

Die Anforderung, bei Schüler/innen Krisen zu induzieren, findet sich dann ausdrücklich bei Helsper (etwa 2014, S. 219). Initiiert werden sollen nicht die in der Sozialisationsinstanz Schule ohnehin zum Tragen kommenden ontogenetischen Ablösungskrisen, sondern sog. „Bildungskrisen“ (ebd.), d.h. Krisen, die sich durch die Auseinandersetzung mit der im Unterricht behandelten Sache ergeben. Kriseninduktion steht dabei in einer Nähe zur Erzeugung von Irritationen, denn es gilt, dass 
Lehrpersonen ,das kognitive Niveau, die erreichten Kompetenzen und Wissensbestände von SchülerInnen immer wieder unter Veränderungsdruck setzen müssen, die erreichten Deutungs- und Erklärungsmuster immer wieder zu irritieren haben“ (Helsper 2000, S. 146). Lehrpersonen haben demnach als „,sachbezogene Kriseninduzierer“ (Helsper 2016, S. 228) zu agieren.

In einer neueren Veröffentlichung legt Helsper (2018) dar, dass es eines spezifischen professionellen Habitus - eines ,wissenschaftlich-reflexiven, forschenden Habitus“ (ebd., S. 129) - bedarf, damit Lehrpersonen durch Kriseninduktion Bildungsprozesse befördern können: „Für diese bildende Eröffnung lebenspraktischer Autonomie in Form von materialen und formalen Bildungsprozessen muss das Lehrerhandeln [...] die bereits entfalteten Selbst- und Weltdeutungen auch immer wieder irritieren und damit Krisen des Wissens auslösen, die als Entstehungsorte des neuen Wissens fungieren können“ (ebd.). Pointiert wird der professionelle Habitus dabei als „Kriseninitiator“ bezeichnet, der ,sich selbst immer wieder der Krise aussetzt“ (ebd., S. 130).

Auch Combe und Gebhard fokussieren die Möglichkeit, dass Lehrpersonen Schülerkrisen im Unterricht induzieren. Durch die Förderung der Arbeit mit ,Vergleichshorizonten“ (Combe und Gebhard 2012, S. 10) können Lehrpersonen anregen, dass Schüler/innen Deutungs- und Erklärungsmuster in Frage stellen. Indem Lehrpersonen Differenzen zwischen den „alltagssprachlichen Zugängen“ von Schüler/innen und den „fachlichen Konzepten“ (Combe und Gebhard 2007, S. 91) nicht ignorieren, sondern Perspektiven gegeneinanderführen, können sie Verstehenskrisen auf Schülerseite induzieren. Unter Rückgriff auf die Philosophie Waldenfels' wird die Verstehenskrise als „Fremdheitszumutung“ gefasst, ,die nicht überwältigt, sondern anregt" (ebd.), d.h. die Schüler/innen zum Nachdenken bringt. Bedeutsam ist dabei mit Blick auf Kriseninduktion, dass Lehrpersonen gemäß den beiden Autoren keine für Schüler/innen spektakulären oder dramatischen Ereignisse herbeiführen müssen. Oftmals genügen ,geringe Verschiebungen und Abweichungen gegenüber dem Erwarteten“ (Combe und Gebhard 2012, S. 61).

Zusammenfassend zeigt sich, dass sich die Thematisierung der Induktion von Schülerkrisen im strukturtheoretischen Ansatz gewandelt hat. Zentral ist nicht mehr die therapeutische Funktion, sondern die Eröffnung sachbezogener Lern- und Bildungsprozesse qua Induktion von Wissens- und Verstehenskrisen. Gleichzeitig erscheint die Induktion von Schülerkrisen weiterhin als Norm bzw. als wesentlicher Teil einer an den Lehrerberuf herangetragenen Anforderungslogik. Die genannten Autoren kommen darin überein, dass Schülerkrisen von Lehrpersonen im Unterricht zwar herbeigeführt werden sollen, aber angesichts dessen, dass sie die Kehrseite von Routinen darstellen, nicht mit Sicherheit herbeigeführt werden können (etwa Oevermann 2002; Helsper 2000; Combe und Gebhard 2012). Kriseninduktion lässt sich demnach selbst als nicht-standardisierbares, krisenhaftes Unterfangen verstehen.

\section{Studien zu Kriseninduktion durch Lehrpersonen}

Die Studienlage zur Induktion von Schülerkrisen durch Lehrpersonen ist überschaubar. Aus schulpädagogischer Perspektive sind insbesondere die Studien der Arbeits- 
gruppe um Gruschka aus dem Projekt „Pädagogische Rekonstruktion des Unterrichtens“" (PAERDU) anzuführen. In den auf Unterrichtstranskripten basierenden Analysen zeigt sich, dass immer wieder Krisen des Nicht-Verstehens auftreten. Schüler/ innen der Sekundarstufe sind dadurch beunruhigt, dass sie in der Begegnung mit der unterrichtlichen Sache erkennen, nicht zu wissen, was sie bereits $\mathrm{zu}$ wissen glaubten (Pollmanns und Gruschka 2013). Diese von Gruschka als Potential für Lernprozesse gedeutete Krisenhaftigkeit wird in der Regel von Lehrpersonen nicht aufgegriffen. Statt die Erschließung der Sache voranzutreiben, werden sinnvoll erscheinende Anschlüsse immer wieder verpasst (etwa Gruschka 2010). Lehrpersonen wehren Schülerkrisen, die den Unterricht „ins Uferlose und Ungesicherte führen“ (ebd., S. 19f.) könnten, in aller Regel ab, etwa durch didaktische Vereinfachungen. Dadurch kommt es zu einem reibungslosen Unterricht, der den Schüller/innen letztlich sinnentleert erscheint.

Die Ergebnisse aus PAERDU decken sich mit anderen rekonstruktiven Studien zu Unterrichtsprozessen und Lehrerhandeln, aus denen hervorgeht, dass im Unterricht „routinisierte Ablaufmuster“ dominieren und Lehrpersonen primär an einem „Fortgang des Unterrichts“ (Helsper 2014, S. 226) arbeiten. Lehrpersonen agieren demnach in der Regel nicht als Kriseninduzierer/innen, sondern als Hüter/innen von Routinen. Dies drückt sich etwa darin aus, dass sie schnelle Bewertungen vornehmen und Ungewissheit nicht aushalten (Combe und Paseka 2012), sachbezogene Schülerfragen als Unterrichtsstörung verstehen und an der Herstellung eines klassenöffentlichen Unterrichts arbeiten (Wenzl 2014) oder verschiedene Muster von Schließungen im Unterricht umsetzen, wodurch Vieldeutigkeiten vereindeutigt und Ungeplantes ausgeschlossen wird (Paseka und Schrittesser 2018).

Weitet man die Perspektive und bezieht auch kommunikations- und konversationsanalytische Studien zu lehrerzentriertem Unterricht ein, zeigt sich, dass Lehrperson in der Regel mittels eines Dreischritts aus Initiation, Vergabe der turns an Schüler/innen und Evaluation der Schülerbeiträge daran arbeiten, einen geregelten Unterrichtsablauf sicherzustellen (etwa Becker-Mrotzek und Vogt 2009). Buttlar (2019) arbeitet dabei mittels Analyse videographierten Grundschulunterrichts drei lehrerseitige Verfahren im Umgang mit Schüleräußerungen heraus, mit denen Lehrpersonen in der Interaktion Angemessenheit herstellen: ,zurückweisende“, ,,akzeptierende“ und „,bearbeitende“ (ebd., S. 105) Verfahren, wobei sich letztgenannte entweder an „Reparaturbedürfigkeit“" oder „Würdigung“ (ebd.) der Schüleräußerung orientieren. Alle Verfahren dienen letztlich dazu, die durch die Lehrperson gesteuerte thematische Progression des Unterrichts zu unterstützen. Buttlars Studie steht dabei beispielhaft für andere videobasierte Studien zu Unterricht, in denen primär solche wiederkehrenden Handlungen von Lehrpersonen herausgearbeitet wurden, die zur Reproduktion bestimmter Praktiken und Interaktionsmuster beitragen (für den Bereich qualitativer Videostudien etwa Rabenstein und Steinwand 2016; für den Bereich quantitativer Videostudien etwa Praetorius et al. 2014). Derartige videobasierte Studien der Unterrichtsforschung stützen den obigen Befund, dass die Induktion von Schülerkrisen als unvorhersehbare, nicht-standardisierbare Tätigkeit eher einen Sonder- denn den Regelfall darstellt.

Erkennbar wird zudem insgesamt, dass Forschung dazu fehlt, wieso Lehrpersonen eher an der Schließung von Kontingenz arbeiten als Krisen von Schüler/innen Raum 
zu geben bzw. unter welchen Bedingungen sie selber als Kriseninduzierer/innen tätig werden. Die eigene Studie setzt an dieser Forschungslücke an.

\section{Forschungsfrage und Studiendesign}

Um näher zu ergründen, unter welchen Bedingungen Lehrpersonen als Kriseninduzierer/innen tätig werden, wurde folgende Forschungsfrage verfolgt:

Welche handlungsleitenden Wissensbestände liegen der Kriseninduktion von Lehrpersonen zugrunde?

Zur Beantwortung dieser Frage wurde auf episodische Interviews (Flick 2010) zurückgegriffen, die mit Lehrpersonen geführt und mit der Dokumentarischen Methode (Bohnsack 2014, 2017) ausgewertet wurden. Aus praxeologisch-wissenssoziologischer Perspektive heraus erscheinen Interviews dann geeignet, Bedingungen für Kriseninduktion zu ergründen, wenn in ihnen ,proponierte Performanz“ (Bohnsack 2017, S. 93) zum Ausdruck kommt, d.h. wenn Lehrpersonen ihre alltägliche Handlungspraxis in Erzählungen und Beschreibungen zur Darstellung bringen. Können Lehrpersonen ihre „Relevanz-, Wissens- und Wertesysteme“ (ebd., S. 328) im Interview entfalten, lässt sich über eine Rekonstruktion der Herstellung proponierter Performanz ein Zugang zu jenem Wissen - verstanden als Orientierungen - gewinnen, das dem beruflichen und damit ggf. auch dem kriseninduzierenden Handeln zugrunde liegt.

Das Datenmaterial - 26 leitfadengestützte Interviews mit Lehrpersonen aus den Jahren 2013 und 2014 - wurde im Rahmen einer Evaluationsstudie (Paseka et al. 2017) an drei Baden-Württemberger Gemeinschaftsschulen erhoben. Die zwischen 44 und 90 minütigen Interviews wurden so angelegt, dass die Lehrpersonen zunächst gebeten wurden, Situationen ihres jeweils zurückliegenden Arbeitstages darzulegen (Interviewteile 1). Erst im weiteren Verlauf wurden Fragen gestellt, die der Evaluation dienten (Interviewteile 2). Die in diesem Artikel fokussierten ersten Interviewteile wurden im Rahmen einer Studie zu Lehrerkrisen im Berufsalltag ausgewertet - einer Studie, in der das Thema der Induktion von Schülerkrisen bereits mitgeführt, aber anders als in diesem Artikel nicht zentral gesetzt wurde (Hinzke 2018).

Den Interviews voraus gingen Shadowings (McDonald und Simpson 2014), in denen jeweils einer Lehrperson einen Tag lang im Rahmen einer nicht-aktiv teilnehmenden Beobachtung gefolgt wurde. Die Shadowings wirkten sich insofern auf die Interviews aus, als die Lehrpersonen relativ detailliert über konkrete Situationen des zurückliegenden Arbeitstages sprechen konnten.

Bei der Analyse der ersten Interviewteile, in denen die Lehrpersonen über Erfahrungen am zurückliegenden Arbeitstag sprechen, erwies sich insbesondere die von Bohnsack (2017) dargelegte Unterscheidung von propositionaler und performativer Logik als weiterführend. Die propositionale Logik verweist auf jenes kommunikative, explizite Wissen, das „Orientierungsschemata“ (ebd., S. 85) und damit u.a. Normen, Rollen und Common Sense-Theorien zugrunde liegt. Die performative Logik hingegen verweist auf konjunktive, implizite Wissensbestände, aus denen „Orientierungsrahmen im engeren Sinne“ (ebd., S. 104) bestehen. Orientierungsrahmen im engeren Sinne setzt Bohnsack dabei mit Habitus gleich und bezieht sie 
auf kollektive Bestände des Erfahrungswissens (ebd.). Propositionale und performative Logik respektive Orientierungsschemata und Orientierungsrahmen im engeren Sinne befinden sich in einer ,notorischen Diskrepanz“ (ebd., S. 106) zueinander, welche von Akteur/innen durch Formen ,implizite[r] Reflexion“ (ebd., S. 107) bearbeitet wird. Die Akteur/innen bilden also eine Praxis aus, mit der Diskrepanz umzugehen, wobei diese Praxis durch einen „Orientierungsrahmen im weiteren Sinne" (ebd., S. 104) strukturiert wird. Dieser Orientierungsrahmen verweist auf solches Erfahrungswissen, das ,auf Grundlage eines gemeinsamen, eines kollektiven oder eben konjunktiven Erlebens der habitualisierten Praxis und ihres Verhältnisses zur Norm“ (ebd., S. 103f.) entsteht. Orientierungsrahmen im weiteren Sinne umgreifen somit die Diskrepanz zwischen propositionaler und performativer Logik respektive zwischen Orientierungsschemata und Orientierungsrahmen im engeren Sinne und strukturieren die implizite Bearbeitung dieser Diskrepanz.

Die Interviewauswertung diente der Eruierung jener Wissensbestände bzw. Orientierungen, die der Arbeit an der Induktion von Schülerkrisen zugrunde liegen. Dazu wurde zunächst mittels formulierender Interpretation das Gesagte paraphrasiert und zu Themen gebündelt. Bei der reflektierenden Interpretation war es wesentlich, eine genaue Textsortentrennung durchzuführen (Nohl 2017, S. 32 ff.). Durch die Einnahme einer genetischen Analyseeinstellung (Bohnsack 2014, S. 58 f.) wurde deutlich, dass sich die performative Logik primär in Beschreibungen und Erzählungen, aber auch in der Art und Weise zeigt, wie die Lehrpersonen Argumentationen und Bewertungen nutzen. Daraufhin wurden im Inter- und Intra-Fallvergleich und unter Beachtung der Sequenzialität der Transkripte positive und negative Gegenhorizonte sowie das sich dokumentierende Enaktierungspotential als „Rahmenkomponenten“ (ebd., S. 139) rekonstruiert. Schülerkrisen wurden dabei im Rahmen der Interpretation als von den Lehrpersonen wahrgenommene Momente bestimmt, in denen auf Schülerseite Routinen zum Ende kommen.

Die Auswertung mündete in der Erstellung einer sinngenetischen Typologie (ebd., S. $200 \mathrm{ff}$.). Die qua Abstraktion gewonnene Basistypik bezieht sich auf den Umgang der Lehrpersonen mit Interaktionssituationen im Berufsalltag, die vier qua Spezifizierung generierten Typen bilden Relationen von Orientierungsschemata, Orientierungsrahmen im engeren Sinne und Orientierungsrahmen im weiteren Sinne ab (Hinzke 2018, S. 215 ff., 365 ff.) - Relationen, die sich für den Umgang mit Schülerkrisen als relevant erweisen.

\section{Ergebnisse}

Eine Arbeit an der Induktion von Schülerkrisen ließ sich lediglich in einem Fall erkennen. In den übrigen Fällen zeigen sich andere Umgangsweisen mit Schülerkrisen, die u.a. in einer Abdunklung und Verhinderung von Schülerkrisen bestehen. Nach einer Darlegung der Umgangsweisen mit Schülerkrisen auf Fallebene (Abschn. 4.1) wird präsentiert, auf welchen typischen Orientierungen die Arbeit an der Induktion von Schülerkrisen basiert (Abschn. 4.2). 


\subsection{Falldarstellungen}

Der Fokus der Darstellung liegt gemäß der Forschungsfrage auf jenem Fall, der an der Kriseninduktion arbeitet (Herr Martin). Zur schärferen Fassung dieser Arbeit werden nach den Prinzipien des maximalen (Frau Dehn) und minimalen Kontrastes (Frau Wulf) punktuell andere Umgangsweisen mit Krisen angeführt, die aus den Schilderungen der Lehrpersonen von Situationen des zurückliegenden Arbeitstages hervorgehen. Vorweg sei angemerkt, dass im Folgenden auch bei Herrn Martin von einer Arbeit an der Induktion von Schülerkrisen und nicht von einem kriseninduzierenden Handeln gesprochen wird, weil aus dem Datenmaterial nicht hervorgeht, ob der Lehrer die anvisierte Kriseninduktion als gelungen wahrnimmt, ob es also aus seiner Sicht zur Induktion von Schülerkrisen kam.

In einer Sequenz mit hohen narrativen Anteilen stellt Herr Martin sein Handeln am Untersuchungstag dar. Während des Projektunterrichts in der fünften Klasse habe er sich zu Schülergruppen gesetzt, um Interesse und Präsenz zu zeigen und Probleme der Schüler/innen während einer Gruppenarbeit zu erfassen (Z. 352-365). Herr Martin fährt fort:

ich bin ja dann zu den Gruppen gelaufen, hab einfach mal zugehört und wenn ich dann gemerkt hab, „oh, das gefällt mir jetzt aber noch nicht, in welche Richtung das läuft“, dann dann hab ich Fragen gestellt. „Oder habt ihr über des schon nachgedacht? Oder warum (1) macht ihr das?" (Hr. Martin, Z. 366-369)

Erkennbar wird erstens, dass das dargestellte Handeln mit einer gewissen Leichtigkeit verbunden ist: Geschildert wird ein aus Lehrersicht unproblematischer, ,einfach" verlaufender Dreischritt von den Gruppen zuhören, aufmerken und Fragen stellen. Zweitens zeigt sich, dass sich Herr Martin als aktiver Protagonist in die unterrichtliche Interaktion bzw. Kommunikation der Schüler/innen einbringt. Seine Intervention in die Gruppe basiert auf einem Wechselspiel von Beobachtung der spezifischen Gruppenarbeit (,hab einfach mal zugehört und wenn ich dann gemerkt hab") und der Enttäuschung eigener Erwartungen, wohin die Gruppenarbeit führen soll (,„oh, das gefällt mir jetzt aber noch nicht, in welche Richtung das läuft“"). Die dargestellte Intervention besteht aus einem Stellen von Fragen, die auf a) eine

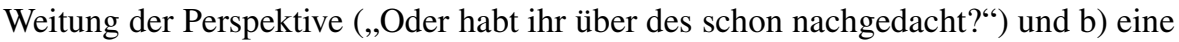
Begründung des Handelns (,Oder warum (1) macht ihr das?“) zielen. Anders ausgedrückt: Herr Martin hinterfragt Entscheidungen der Schüler/innen und sensibilisiert diese für Kontingenzen, d.h. dafür, dass auch andere Optionen existieren.

Im Fallvergleich wird deutlich, dass andere Lehrpersonen auf Schließungen setzen, wenn sie wie Herr Martin ein Schülerverhalten wahrnehmen, das nicht ihren Erwartungen entspricht. Exemplarisch sei das Agieren von Frau Dehn angeführt, die in Bezug auf ihren Kunstunterricht äußert:

Und dann kommt sie halt alle fünf Minuten, [spricht außergewöhnlich schnell] ,ja, ich bin jetz fertig, bin jetz fertig, kann ich jetz Freiarbeit machen?“" Ich so, ,nee, da muschst des und des und des und so“. Also da musst ich immer wieder auch bei ihr so so schieben und ähm dass sie des halt, ja, dass sie da dran bleibt einfach an dem Thema. //mhm// Und des is jetz echt gut geworden, 
das Bild, aber des war halt wirklich immer mit (1) mit Nachschieben so (Fr.

Dehn, Z. 89-95).

Statt der Schülerin einen Anreiz zu geben, über den sie nachdenken könnte, macht die Lehrerin ihr unvermittelt Vorgaben, was sie tun muss. Die Metapher des Schiebens ist dabei mit einer Objektivierung der Schülerin verbunden, die gleich einem Gegenstand von a nach b bewegt wird. Demgegenüber steuert Herr Martin indirekter, indem er durch seine Fragen Kontingenzen aufzeigt und es dadurch zunächst den Schüler/innen überlässt, ob sie ihre Arbeitsweise ändern.

Im Fortgang der obigen Interviewsequenz konkretisiert Herr Martin sein Vorgehen im Projektunterricht. Eine Schülergruppe war seiner Wahrnehmung nach am Untersuchungstag dabei, das Thema „Circus Maximus“ handlungsorientiert zu bearbeiten, indem sie ein Modell des Circus nachbauen wollte. In der folgenden Sequenz wird deutlich, dass der Lehrer seine Intervention vor dem Hintergrund einer schulischen Sach- und Bewertungslogik rechtfertigt.

Und ähm (2) dann ham wir vielleicht nach äh vier Wochen ham die $n \mathrm{n}$ halben Circus gebaut, ham aber gar keine Ahnung, um was es geht. //mhm// Aber sie ham den halben Circus gebaut mit $m$ riesen Engagement. Und da muss ich ihnen sagen, „Tut mir Leid, roter Punkt. War gar nix.“ (2) Na, große Frustration. Also muss ich im Vorfeld ihnen ganz klar machen, da sind schon Erwartungshaltungen. Ich möchte mehr haben von dem Projekt. Ich möchte Hintergrundwissen. Ich möchte dann auch so was wie Brot und Spiele. //mhm// Is n Schlagwort, das gehört da einfach mit dazu (Hr. Martin, Z. 381-388).

Eine rein handlungspraktische Auseinandersetzung mit dem Thema hat eine bestimmte Folge, nämlich eine schlechte Bewertung, was wiederum mit Frustration auf Schülerseite einhergeht. Um dieses im negativen Gegenhorizont stehende imaginierte Szenario zu umgehen, bedürfe es einer Intervention des Lehrers. Die angesprochenen „Erwartungshaltungen“, die sich auf eine kognitive Durchdringung des Unterrichtsthemas beziehen und den positiven Gegenhorizont bilden, werden dabei von Herrn Martin verbürgt, wie sich in der Anapher des dreifachen Satzanfangs mit „Ich möchte“ zeigt. Es deutet sich dabei an, dass dieses Streben nach kognitiver Durchdringung nicht auf willkürlichen Vorlieben des Lehrers basiert, sondern in einem Sachbezug verankert ist, der von Herrn Martin eingefordert wird. Erkennbar wird dies in der Bezeichnung des „Schlagwortes“ „Brot und Spiele“ als etwas, das „da einfach mit dazu“ gehört.

Im Fallvergleich zeigt sich, dass andere Lehrpersonen nicht nur - wie Herr Martin - den unterrichtlichen Sachbezug fokussieren, sondern darüber hinaus auch den familiären Kontext von Schüler/innen in den Blick nehmen. So antizipiert etwa Frau Wulf, dass ihre Schülerin Bianca in diesem Kontext eine Krise erfahren könnte. Die Lehrerin erzählt von einem Gespräch, das sie am Untersuchungstag mit Bianca in Reaktion darauf geführt hat, dass diese von einer anderen Lehrerin eine rote Karte erhalten hatte, woraufhin sie nun eigentlich einen Elternbrief schreiben müsste. Frau Wulf schildert, dass Bianca 
aus nem unheimlich schweren Elternhaus kommt zu Hause und wo eigentlich gerade so dieses sich selber zu reflektieren eigentlich überhaupt nich da is sozusagen. Und von daher war das für mich $\mathrm{n}$ unheimlich schönes Erlebnis heute, dass sie da sehr überlegt eigentlich mir erzählen konnte, was passiert zu Hause und eben, da gings ja um die Unterschrift, ich dann gesagt hab, ,okay, ich kann mit mir reden lassen, dass wir tatsächlich diese Unterschrift nicht brauchen vom Vater", aber dann möcht ich eben wissen, was sie dafür tut (Fr. Wulf, Z. 325-331).

Die Lehrerin rekurriert auf Hintergrundwissen, das ihr über den familiären Kontext ihrer Schülerin vorliegt (,aus nem unheimlich schweren Elternhaus kommt [...]“). Die dortigen Verhältnisse stehen im Kontrast zum eigenen, auf den schulischen Kontext bezogenen positiven Gegenhorizont einer auf Reflexion beruhenden Kommunikation, im Zuge derer die Lehrerin (noch) mehr über den familiären Kontext der Schülerin erfährt (,sehr überlegt eigentlich mir erzählen konnte, was passiert zu Hause"). Angesichts dieses Hintergrundwissens eröffnet Frau Wulf der Schülerin die Möglichkeit, durch Erbringung einer Ersatzleistung eine antizipierte Krise, die bei Einholung der Unterschrift durch den Vater droht, zu umgehen. Aus der weiteren Interviewanalyse geht hervor, dass die Lehrerin dieses krisenverhindernde Handeln zugleich als Chance dafür sieht, dass die Schülerin mit ihr in den Diskurs tritt und Verantwortung für sich übernimmt.

Wiederum wie Frau Wulf sucht auch Herr Martin das Gespräch mit den Schülern. So beschreibt er seine Intervention in die oben genannte Schülergruppe wie folgt:

Ich hab zuerst zwei Fragen gestellt, drei Fragen, die in die Richtung gingen: „Ja warum? Was isch Unterhaltung? Warum isch das wichtig?“ Ja? Hätt ichs dabei belassen sollen? Fragezeichen. Ich weiß es nicht. Wären sie in der nächsten Stunde selber drauf kommen? Weiß ich nicht. //mhm// Die sind so vernarrt, „ein Meter auf ein Meter. Ich bau mein Kolosseum, nein, mein Maximus“, ja? „Und äh mal mit Styropor“ und „oh, mein Opa, der hilft mir“, ja? (Z. 390-396).

Der Lehrer hinterfragt hier, ob seine Entscheidung pro Stichwortsetzung die richtige war, und kann dies nicht endgültig beantworten. Trotz aller konkreten Beobachtungen von Schüler/innen - die Wiedergabe der wörtlichen Zitate spricht dafür - ist es Herrn Martin nicht möglich, das künftige Handeln seiner Schüler/innen vorherzusehen.

Hier bietet sich erneut eine Kontrastierung mit Frau Dehn an, die nicht situationsspezifische, sondern generalisierende Aussagen trifft, in diesem Fall über einen Schüler, der ihrer Ansicht nach im Kunstunterricht nicht mitgearbeitet hat:

Ich hab ihn einmal in der Woche, äh ich kenn seine Familie nich. [...] er is einfach ein unkonzentriertes Kind (Fr. Dehn, Z. 183-186).

Im Gegensatz zu Frau Wulf arbeitet die Lehrerin nicht daran, mehr über die familiären Hintergründe ihrer Schüler/innen zu erfahren. Stattdessen liefert sie eine Erklärung, die als Zuschreibung auf die Gesamtperson des Schülers gedeutet werden kann (,er is einfach ein unkonzentriertes Kind“). Etwaige Krisen, die hinter dem 
die Erwartungen der Lehrerin enttäuschenden Schülerverhalten stehen, werden wie oben in der Situation mit der Kunstschülerin nicht eruiert, sondern abgedunkelt.

Bei Herrn Martin wird indes im weiteren Verlauf des Interviews abermals erkennbar, dass er eine schulische Sach- und Bewertungslogik mitdenkt:

Dass von dem Projekt als solches ne Wichtigkeit, weils ja auch nen ne Grundlage da sein soll, dass des aus- außer Acht gelassen wird. Und ich denke, dass ich in der fünften Klasse durchaus da noch Hilfestellungen geben kann. Wenn wir nachher Neuntklässler haben, die bei ihrer Projektprüfung da sind, wäre das nicht möglich. //mhm// Aber wie sollen sies lernen? (2) Man muss ihnen jetzt ja sagen, ,,schön, dass du was bauen willst. Schön, dass ich was seh. Aber das allein isch es nicht“. Also hab ich mich für das entschlossen, nach zwei, drei Mal nachfragen, [atmet aus] sie aber nicht wirklich so in diese Richtung tendiert haben, dass ich einfach mal gesagt hab, „Hey, da gibts n Schlagwort. Guckt mal, was bedeutet des?“ (Hr. Martin, Z. 396-406).

Der Lehrer erklärt sein Handeln vor dem Hintergrund einer Differenzierung von Klassenstufen in der Organisation Schule. Während Neuntklässler/innen wesentlich einer Bewertungslogik unterliegen, haben Fünftklässler/innen noch die Möglichkeit zu ,lernen“. Herr Martin positioniert sich hier - anders als Frau Dehn - als jemand, der für solche Lernprozesse mitverantwortlich ist und argumentiert, dass es für ein solches sachbezogenes Lernen neben Bestätigung auch einer Kontrastfolie bedarf, die mit der Routine der Schüler/innen im Umgang mit Unterrichtsgegenständen bricht („Aber das allein isch es nicht“). Sein Handeln am Untersuchungstag stellt der Lehrer in eine Linie mit diesen Überlegungen: Nach zwei bis drei Nachfragen, die nicht die erhoffte Folge zeitigten, habe er ein zweites Mal interveniert, der Gruppe ein Schlagwort - „Brot und Spiele“ - genannt und sie aufgefordert, dessen Bedeutung zu eruieren.

Insgesamt betrachtet lässt sich die Vorgehensweise von Herrn Martin als Arbeit an einer sachbezogenen Kriseninduktion interpretieren. Auf Grundlage konkreter Beobachtungen von Unterrichtssituationen sowie bestimmter Vorstellungen über eine Sach- und Bewertungslogik im schulischen Raum gibt Herr Martin - anders als Frau Dehn (,Schieben“) - den Schülern keine eindeutigen Handlungsvorschriften, sondern eröffnet ihnen - ähnlich wie Frau Wulf (,,möcht ich eben wissen, was sie dafür tut") - durch die Formulierung von Fragen Gedanken- und Möglichkeitsräume. Erst in einem zweiten Schritt fordert er die Schüler auf, sich mit einem Schlagwort auseinanderzusetzen, dessen Bedeutung er offen lässt. Anders als bei Frau Wulf dokumentiert sich bei Herrn Martin keine Prävention möglicher Schülerkrisen. Durch das Aufzeigen von Alternativen ermöglicht der Lehrer hingegen potentiell Krisen auf Schülerseite in dem Sinne, dass die Schüler möglicherweise beginnen, ihr Handeln zu hinterfragen.

\subsection{Verortung der Arbeit an der Kriseninduktion in typischen handlungsleitenden Orientierungen}

Der bei Herrn Martin analysierten Arbeit an der Kriseninduktion liegt eine Orientierungsstruktur zugrunde, die einen von vier rekonstruierten Typen der Relation 
von Orientierungsschemata, Orientierungsrahmen im engeren und Orientierungsrahmen im weiteren Sinne abbildet (Hinzke 2018, S. $367 \mathrm{ff}$.). Demnach arbeitet sich Herr Martin als Repräsentant des Typus „Rekonstruktive Suche innerhalb einer offenen Entscheidungspraxis“ auf der Ebene der Orientierungsschemata an den Erwartungen ab, dass Lehrpersonen allen Schüler/innen gerecht werden und in ihrem Berufsalltag stets Entscheidungen treffen müssen, wobei sie der Sach- und Bewertungslogik von Schule nachkommen müssen. Diese Normen und Common SenseTheorien stehen in einer Spannung zum Orientierungsrahmen im engeren Sinne, der darin besteht, Entscheidungen zu treffen, die den Verlauf der unterrichtlichen Interaktion und Kommunikation mit Schüler/innen bedingen. Die Spannung zwischen den wahrgenommenen normativen Erwartungen und der Entscheidungspraxis bearbeitet Herr Martin auf Basis einer suchend-erschließenden Beobachtungshaltung als Ausdruck eines Orientierungsrahmens im weiteren Sinne. Dieser zeigt sich im Sammeln konkreter Informationen über Situationen und Schüler/innen, im Treffen von Entscheidungen, im Bestreben der Absicherung dieser Entscheidungen durch Informationen und im Vermögen, eine distanzierte Sicht auf die eigene Praxis einzunehmen.

Im Unterschied zu dieser Beobachtungshaltung dunkeln Lehrpersonen mit einer subsumtiv-generalisierenden Beobachtungshaltung - der Fall Frau Dehn steht für diesen maximalen Kontrast - mögliche Schülerkrisen dadurch ab, dass diese in ihrem Zustandekommen und ihrer Art nicht ergründet werden, sondern als etwas gefasst werden, das es mittels der Kommunikation von Vorgaben an Schüler/innen schnell zu beseitigen gilt.

Die suchend-erschließende Beobachtungshaltung stellt aber nicht die einzige Voraussetzung für ein auf Kriseninduktion ausgerichtetes Handeln dar, denn nicht alle Lehrpersonen mit dieser Beobachtungshaltung handeln derart, wie sich im Fall Frau Wulf als minimalem Kontrast zeigt. Die These lautet, dass die Arbeit an einer sachbezogenen Kriseninduktion einer suchend-erschließenden Beobachtungshaltung bedarf, die sich in der Bearbeitung der Spannung zwischen der Wahrnehmung einer Sach- und Bewertungslogik in der Organisation Schule und der habituellen Etablierung einer Entscheidungspraxis im Unterricht entfaltet.

Lehrpersonen, die an der Induktion von Schülerkrisen arbeiten, müssen - so die Weiterführung der These - über ein Selbstverständnis verfügen, das es ihnen erlaubt, sich selbst als wirksame Akteur/innen im Unterricht mit Handlungsoptionen zu erleben. Die Wahrnehmung schulbezogener Logiken führt hier nicht primär zum Erleben einschränkender Fremdbestimmung, sondern treibt dazu an, sachbezogene Lernprozesse bei Schüler/innen zu initiieren. Schüler/innen geraten als Lernende in den Blick und der Unterricht erscheint als durch Kontingenz strukturiertes, Ungewissheit erzeugendes System. Folglich werden mögliche Schülerkrisen als Chance für Lernprozesse angesehen - ohne dass sichergestellt werden kann, dass Lernprozesse einsetzen. Genau diese Unsicherheit und der damit einhergehende Entscheidungsdruck tragen dazu bei, dass Herr Martin wiederum eigene Krisen erfährt (Hinzke 2018, S. $290 \mathrm{ff}$.). 


\section{Diskussion}

Die generierten Ergebnisse lassen erkennen, dass sich die Nutzung der Dokumentarischen Methode als gewinnbringend für die interpretative Erschließung einer Arbeit an der Kriseninduktion erwiesen hat. So bietet die Studie einen Hinweis darauf, wie erklärt werden kann, dass Lehrpersonen im Unterricht tendenziell eher an der SchlieBung denn an der Induktion von Schülerkrisen arbeiten (s. Kap. 2). Kriseninduktion erscheint als voraussetzungsvoll, da sie auf eine komplexe, spezifische Relation von Normen und Common Sense-Theorien sowie Orientierungsrahmen im engeren und im weiteren Sinne zurückzuführen ist. Es bedarf demnach bestimmter Erfahrungsund Wissensaufschichtungen, die es ermöglichen, dass Lehrpersonen an der Induktion von Schülerkrisen arbeiten, ohne dass der Erfolg dieses Handelns sichergestellt ist.

Eine Limitation der Studie besteht in der relativ kleinen Fallzahl, was Aussagen über die Tragweite der Ergebnisse erschwert. Weitere Forschung unter Einbezug anderer Schulformen und Jahrgangsstufen sowie der Rekonstruktion der Interaktionsstruktur von Unterricht, d.h. der ,performativen Performanz“ (Bohnsack 2017, S. 93), etwa qua Audio- oder Videographien, würden zur weiteren Erschließung des Themas beitragen. Generalisierungspotential zeigt sich hingegen mit Blick auf aktuelle Studien, in denen sich die Relationierung von propositionaler und performativer Logik ebenfalls als fruchtbar für die Erschließung von Lehrerhandeln herausgestellt hat (etwa Hericks et al. 2018).

Aus strukturtheoretischer Perspektive lässt sich die rekonstruierte Arbeit an der Kriseninduktion (Hr. Martin) als professionalisiertes Handeln deuten. So richtet sich diese Arbeit an der Sache des Unterrichts und den ,erreichten Kompetenzen und Wissensbestände[n]“ der Schüler/innen aus und setzt diese ,unter Veränderungsdruck“ (Helsper 2000, S. 146). Dabei nutzt Herr Martin über das Einbringen eines Konzepts (,Stichwort“ Brot und Spiele), das den Schüler/innen seiner Wahrnehmung nach nicht vertraut ist, das Potenzial einer Arbeit mit ,Vergleichshorizonten“ (Combe und Gebhard 2012, S. 10). Zudem reflektiert der Lehrer über die Nicht-Standardisierbarkeit und die ungewissen Folgen seines Vorgehens. Dagegen ist die Abdunklung von Schülerkrisen (Fr. Dehn) bei gleichzeitigem nicht erkennbaren „Fallverstehen“ (Helsper 2000, S. 145) als nicht professionalisiert zu bewerten. Professionalisiertes Handeln würde stattdessen im Aufbau eines ,Arbeitsbündnisses“ als ,widersprüchliche[r] Einheit von spezifischen und diffusen Sozialbeziehungen“ (Oevermann 1996, S. 148) sowohl mit den jeweiligen Lerngruppen als auch mit Einzelschüler/innen bestehen. Bei der Verhinderung von antizipierten, möglicherweise auf die Person von Schüler/innen ausgreifenden Krisen, die über den Unterricht hinaus in die familiäre Sphäre wirken könnten, wäre zu diskutieren, inwiefern hier professionalisiertes Handeln vorliegt - insbesondere dann, wenn wie im dargelegten Fall (Fr. Wulf) negative Folgen verhindert werden sollen und verlangt wird, dass die Schüler/innen alternative Lösungen finden, die für sie Neues hervorbringen. Relevant wird hier die von Helsper (2000) ausgearbeitete „Näheantinomie“ (ebd., S. 147), denn die Lehrerin greift über die schulische Sphäre hinaus auf die Privatsphäre der Schülerin aus, was einerseits Nähe erfordert, andererseits eine „distanzierende Begrenzung erzwingt“" (ebd., S. 148). Mit Wernet (2003) lässt sich bei diesem Vorgehen auch 
nach einer „Entgrenzung“ (ebd., S. 7) des Lehrerhandelns fragen, da nicht ersichtlich wird, dass Frau Wulf eine „universalistisch-unpersönliche[n] Leistungsorientierung“ (ebd., S. 115) durchzusetzen sucht.

Aus konversationsanalytischer Perspektive betrachtet müsste das unterrichtliche Kommunikationssetting beachtet werden, in dem die Lehrpersonen mit Schülerkrisen umgehen. Es stellt sich die Frage, ob die besondere Form der Gruppenarbeit, wie sie im Unterricht von Herrn Martin vorliegt, die Arbeit an der Kriseninduktion unterstützt bzw. sogar erst ermöglicht, während im lehrergelenkten, klassenöffentlichen Unterricht, wie er sich im Fall insbesondere von Frau Dehn zeigt, ggf. Zugzwänge wirksam werden könnten, die einer Arbeit an der Kriseninduktion eher im Wege stehen (Buttlar 2019; auch Wenzl 2014).

Was könnte Lehrerprofessionalität nun in einer praxeologisch-wissenssoziologischen Perspektive bedeuten? Bohnsack $(2017,2020)$ hat sich in letzter Zeit mit genau dieser Frage auseinandergesetzt. Bezogen auf das in diesem Beitrag fokussierte Thema wäre eine Kriseninduktion dann als professionalisiert zu bezeichnen, wenn das entsprechende Handeln konstitutive Bedingungen von Professionalität sowie diskursethische Prinzipien erfüllt, die beide aus der Rekonstruktion einer ,praktischen Klugheit" (Bohnsack 2017, S. 329) hervorgehen und damit prinzipiell veränderbar sind. Kriseninduktion stellt dabei eine von mehreren möglichen Ausdrucksformen professionalisierten Handelns dar. Entscheidend ist, ob es Lehrpersonen gelingt, in der Interaktion mit Schüler/innen eine sog. ,konstituierende Rahmung“ (Bohnsack 2020, S. 31) hervorzubringen und aufrechtzuerhalten. Damit ist eine Prozessstruktur gemeint, die sich daraus ergibt, dass berufliche Akteur/innen ,im Zuge der Bewältigung der notorischen Diskrepanz zwischen [...] komplexen normativen Anforderungen der Organisation und der Konstitution einer gemeinsamen Praxis mit der Klientel im Sinne eines konjunktiven Erfahrungsraums“ (ebd.) ,interaktiv-routiniert“ (ebd.) Entscheidungen treffen. Die konstituierende Rahmung ermöglicht somit, organisationale Programme in einen konjunktiven Erfahrungsraum, d.h. in Räume des unmittelbaren Einander-Verstehens (ebd., S. 28), zu integrieren, was bedeutet, dass Entscheidungen an Entscheidungen anknüpfen (ebd., S. 32), sich also im Fall des Lehrerberufs Routinen in der schulischen bzw. unterrichtlichen Interaktion zwischen Lehrpersonen und Schüler/innen ausbilden können. Somit müsste geprüft werden, ob Lehrpersonen durch die Induktion von Schülerkrisen an der Herstellung einer konstituierenden Rahmung arbeiten, die anschlussfähig an die in der Organisation Schule und im konjunktiven Erfahrungsraum mit den Schüler/innen geteilten Logiken ist. Dies wäre beispielsweise nicht der Fall, wenn die Kriseninduktion an der Sachlogik von Schule vorbei primär auf die Schüler/innen ,als ganze Person[en]“ (Oevermann 1996, S. 105) zielen und in deren persönliche Integrität eingreifen würde (Bohnsack 2020, S. 27). Eine Arbeit an einer Kriseninduktion indes, die ausschnitthaft an dem Wissen und den Reflexionspotentialen von Schüler/innen ansetzt, indem sie - wie es etwa aus den Erzählungen von Herrn Martin hervorgeht - auf fallspezifischen Beobachtungen von Schüler/innen beruht und zugleich organisationale Logiken von Schule zur Geltung zu bringen sucht, erscheint demgegenüber als professionalisiertes Handeln. Dies gilt insbesondere, wenn die Lehrpersonen wie es sich ebenfalls bei Herrn Martin dokumentiert - über ihr intervenierendes 
Handeln und die letztendliche Unvorhersehbarkeit der Folgen einer sachbezogenen Kriseninduktion reflektieren.

Weitere Forschung wäre angesichts der generierten Ergebnisse zur Frage nötig, ob die Arbeit an sachbezogener Kriseninduktion zwingend eine suchend-erschließende Beobachtungshaltung als Orientierungsrahmen im weiteren Sinne voraussetzt, die sich als Resultat der Spannung zwischen bestimmten theoretischen (Sach- und Bewertungslogik) und habituellen Wissensbeständen (Etablierung einer Entscheidungspraxis im Unterricht) entfaltet, oder ob auch andere Konstellationen von Wissensbeständen zugrunde liegen können. Auch wäre zu erforschen, worin sich eine erfolgreiche Kriseninduktion zeigt und wie Lehrpersonen Schüler/innen nach einer Kriseninduktion bei der Bewältigung der induzierten Krisen unterstützen können.

Mit Blick auf Lehrer/innenbildung wäre zu erforschen, welchen Beitrag deren drei Phasen zu einer professionalisierten Kriseninduktion leisten können. Anders als Lehrpersonen stehen etwa Studierende nicht in der Verantwortung, in der Interaktion mit Schüler/innen eine konstituierende Rahmung zur Geltung zur verhelfen. Allerdings könnte universitäre Lehrer/innenbildung insofern auf eine professionalisierte Kriseninduktion und einen reflexiven Umgang damit vorbereiten, als sie bei den Studierenden vorhandene „Reflexionspotentiale“ (Bohnsack 2020, S. 95) und „Analyseeinstellungen“ (ebd., S. 17) in den Blick nimmt und aufbauend darauf zur Perspektiverweiterung beizutragen sucht. Wodurch eine solche Perspektiverweiterung im Studium allerdings ermöglicht werden könnte und wie nachhaltig sie auch nach Abschluss des Studiums im Referendariat und im Berufsalltag wirkt, stellen weitgehend offene Forschungsfragen dar.

Funding Open Access funding provided by Projekt DEAL.

Open Access Dieser Artikel wird unter der Creative Commons Namensnennung 4.0 International Lizenz veröffentlicht, welche die Nutzung, Vervielfältigung, Bearbeitung, Verbreitung und Wiedergabe in jeglichem Medium und Format erlaubt, sofern Sie den/die ursprünglichen Autor(en) und die Quelle ordnungsgemäß nennen, einen Link zur Creative Commons Lizenz beifügen und angeben, ob Änderungen vorgenommen wurden.

Die in diesem Artikel enthaltenen Bilder und sonstiges Drittmaterial unterliegen ebenfalls der genannten Creative Commons Lizenz, sofern sich aus der Abbildungslegende nichts anderes ergibt. Sofern das betreffende Material nicht unter der genannten Creative Commons Lizenz steht und die betreffende Handlung nicht nach gesetzlichen Vorschriften erlaubt ist, ist für die oben aufgeführten Weiterverwendungen des Materials die Einwilligung des jeweiligen Rechteinhabers einzuholen.

Weitere Details zur Lizenz entnehmen Sie bitte der Lizenzinformation auf http://creativecommons.org/ licenses/by/4.0/deed.de.

\section{Literatur}

Becker-Mrotzek, M., \& Vogt, R. (2009). Unterrichtskommunikation. Linguistische Analysemethoden und Forschungsergebnisse (2. Aufl.). Tübingen: Niemeyer.

Bohnsack, R. (2014). Rekonstruktive Sozialforschung. Einfuihrung in qualitative Methoden (9. Aufl.). Opladen: Budrich.

Bohnsack, R. (2017). Praxeologische Wissenssoziologie. Opladen: Budrich.

Bohnsack, R. (2020). Professionalisierung in praxeologischer Perspektive. Opladen: Budrich. 
Bonnet, A., \& Hericks, U. (2014). Professionalisierung und Deprofessionalisierung im Lehrer/innenberuf. Zeitschrift für interpretative Schul- und Unterrichtsforschung, 3, 3-8.

Buttlar, A.-C. (2019). Sequenzielle Analysen interaktiver Verfahren des lehrerseitigen Umgangs mit Schüleräußerungen. In K. Verrière \& L. Schäfer (Hrsg.), Interaktion im Klassenzimmer (S. 97-117). Wiesbaden: Springer.

Combe, A., \& Gebhard, U. (2007). Sinn und Erfahrung. Zum Verständnis fachlicher Lernprozesse in der Schule. Opladen: Budrich.

Combe, A., \& Gebhard, U. (2012). Verstehen im Unterricht. Zur Rolle von Phantasie und Erfahrung. Wiesbaden: Springer VS.

Combe, A., \& Paseka, A. (2012). Und sie bewegt sich doch? Zeitschrift für Bildungsforschung, 2(2), 91-107.

Combe, A., Paseka, A., \& Keller-Schneider, M. (2018). Ungewissheitsdynamiken des Lehrerhandelns. In A. Paseka, M. Keller-Schneider \& A. Combe (Hrsg.), Ungewissheit als Herausforderung für pädagogisches Handeln (S. 53-79). Wiesbaden: Springer VS.

Flick, U. (2010). Qualitative Sozialforschung. Eine Einführung (3. Aufl.). Reinbek: Rowohlt.

Gruschka, A. (2010). An den Grenzen des Unterrichts. Opladen: Budrich.

Helsper, W. (2000). Antinomien des Lehrerhandelns und die Bedeutung der Fallrekonstruktion. In E. Cloer, D. Klika \& H. Kunert (Hrsg.), Welche Lehrer braucht das Land? (S. 142-177). Weinheim: Juventa.

Helsper, W. (2014). Lehrerprofessionalität - der strukturtheoretische Professionsansatz zum Lehrberuf. In E. Terhart, H. Bennewitz \& M. Rothland (Hrsg.), Handbuch der Forschung zum Lehrerberuf (2. Aufl. S. 216-240). Münster: Waxmann.

Helsper, W. (2016). Pädagogische Lehrerprofessionalität in der Transformation der Schulstruktur. In T.S. Idel, F. Dietrich, K. Kunze, K. Rabenstein \& A. Schütz (Hrsg.), Professionsentwicklung und Schulstrukturreform (S. 217-245). Bad Heilbrunn: Klinkhardt.

Helsper, W. (2018). Lehrerhabitus. In A. Paseka, M. Keller-Schneider \& A. Combe (Hrsg.), Ungewissheit als Herausforderung für pädagogisches Handeln (S. 105-140). Wiesbaden: Springer VS.

Hericks, U., Sotzek, J., Rauschenberg, A., Wittek, D., \& Keller-Schneider, M. (2018). Habitus und Normen im Berufseinstieg von Lehrer*innen. Zeitschrift für interpretative Schul- und Unterrichtsforschung, 7, 65-80.

Hinzke, J.-H. (2018). Lehrerkrisen im Berufsalltag. Zum Umgang mit Spannungen zwischen Normen und Orientierungsrahmen. Wiesbaden: Springer VS.

Koller, H.-C. (2012). Bildung anders denken. Einführung in die Theorie transformatorischer Bildungsprozesse. Stuttgart: Kohlhammer.

Kunter, M., Baumert, J., Blum, W., Klusmann, U., Krauss, S., \& Neubrand, M. (Hrsg.). (2011). Professionelle Kompetenz von Lehrkräften. Ergebnisse des Forschungsprogramms COACTIV. Münster: Waxmann.

McDonald, S., \& Simpson, B. (2014). Shadowing research in organizations: the methodological debates. Qualitative Research in Organizations and Management, 9(1), 3-20.

Nohl, A.-M. (2017). Interview und Dokumentarische Methode. Anleitungen für die Forschungspraxis (5. Aufl.). Wiesbaden: Springer VS.

Oevermann, U. (1991). Genetischer Strukturalismus und das sozialwissenschaftliche Problem der Erklärung der Entstehung des Neuen. In S. Müller-Doohm (Hrsg.), Jenseits der Utopie. Theoriekritik der Gegenwart (S. 267-336). Frankfurt a.M.: Suhrkamp.

Oevermann, U. (1996). Theoretische Skizze einer revidierten Theorie professionalisierten Handelns. In A. Combe \& W. Helsper (Hrsg.), Pädagogische Professionalität (S. 70-182). Frankfurt a.M.: Suhrkamp.

Oevermann, U. (2002). Professionalisierungsbedürftigkeit und Professionalisiertheit pädagogischen Handelns. In M. Kraul, W. Marotzki \& C. Schweppe (Hrsg.), Biographie und Profession (S. 19-63). Bad Heilbrunn: Klinkhardt.

Paseka, A., \& Schrittesser, I. (2018). Muster von Schließungen im Unterricht. In A. Paseka, M. Keller-Schneider \& A. Combe (Hrsg.), Ungewissheit als Herausforderung für pädagogisches Handeln (S. 31-52). Wiesbaden: Springer VS.

Paseka, A., Hinzke, J.-H., \& Maleyka, K. (2017). SHIP. Projektbericht

Pollmanns, M., \& Gruschka, A. (2013). Bildung als empirische statt als bloß normative Grundkategorie der Unterrichtsforschung. In K. Müller-Roselius \& U. Hericks (Hrsg.), Bildung (S. 55-83). Opladen: Budrich.

Praetorius, A.-K., Pauli, C., Reusser, K., Rakoczy, K., \& Klieme, E. (2014). One lesson is all you need? Learning and instruction, 31, 2-12. 
Rabenstein, K., \& Steinwand, J. (2016). Praktiken der Differenz(re)produktion im individualisierten Unterricht. In U. Rauin, M. Herrle \& T. Engartner (Hrsg.), Videoanalysen in der Unterrichtsforschung (S. 242-262). Weinheim: Beltz Juventa.

Terhart, E. (2011). Lehrerberuf und Professionalität. Zeitschrift für Pädagogik, 57, 202-224.

Wenzl, T. (2014). Elementarstrukturen unterrichtlicher Interaktion. Zum Vermittlungszusammenhang von Sozialisation und Bildung im schulischen Unterricht. Wiesbaden: Springer VS.

Wernet, A. (2003). Pädagogische Permissivität. Schulische Sozialisation und pädagogisches Handeln jenseits der Professionalisierungsfrage. Opladen: Leske + Budrich. 